\title{
Directional Discrete Cosine Transform for Handwritten Script Identification
}

\author{
Mallikarjun Hangarge ${ }^{\dagger}$, K.C. Santosh ${ }^{\ddagger}$, Rajmohan Pardeshi ${ }^{\dagger}$ \\ ${ }^{\dagger}$ Department of Computer Science \\ Karnatak Arts, Science \& Commerce College, Bidar, INDIA \\ ‡LORIA - Université de Lorraine \\ BP - 239, Loria Campus Scientifique, FRANCE \\ Email: mhangarge@yahoo.co.in, Santosh.KC@loria.fr, rajkascceieee.org
}

\begin{abstract}
This paper presents directional discrete cosine transforms (D-DCT) based word level handwritten script identification. The conventional discrete cosine transform (DCT) emphasizes vertical and horizontal energies of an image and de-emphasizes directional edge information, which of course plays a significant role in shape analysis problem, in particular. Conventional DCT however, is not efficient in characterizing the images where directional edges are dominant. In this paper, we investigate two different methods to capture directional edge information, one by performing 1D-DCT along left and right diagonals of an image, and another by decomposing 2D-DCT coefficients in left and right diagonals. The mean and standard deviations of left and right diagonals of DCT coefficients are computed and are used for the classification of words using linear discriminant analysis (LDA) and $K$-nearest neighbour $(K$ $\mathrm{NN}$ ). We validate the method over 9000 words belonging to six different scripts. The classification of words is performed at biscripts, tri-scripts and multi-scripts scenarios and accomplished the identification accuracies respectively as $96.95 \%, 96.42 \%$ and $85.77 \%$ in average.
\end{abstract}

\section{INTRODUCTION}

Optical character recognition (OCR) is one of the challenging areas of research in pattern recognition, which is basically script dependent. Therefore, to facilitate the OCR process, script separation is necessary. Unlike the other scripts, in context of Indian documents, for instance, we come across with multi-script documents such as bank cheques, application forms and railway reservation forms. An example of bi-script document is shown in Fig. 1. In this paper, we are primarily focused on handwritten script identification. It is basically defined as an automatic identification of different script zones in a text document. It can be done at paragraph, line or word level. Word level script identification is relatively significant than paragraph or line level. This task is simpler when we deal with printed documents, whereas it is harder in case of handwritten documents because of the unconstrained character sizes, gaps between words, lines, writing style, etc. With these factors, we have motivated to outline a generic framework to deal with both printed and handwritten script identification at bottom level i.e., word level.

Over the last three decades, besides the work on printed text, few works are reported on handwritten text script identification of Indic scripts. Almost all the methods either employed global or local and combinations of both global and local features. Primarily, global features used in [1]-[6] are based on DCT, DWT, Gabor, steerable pyramids, and the Radon transform. The local features, for example, shape features of connected components are employed in [7]-[12]. The combination of these are employed in [13] and called it as global method. A detailed review of literature of script identification is presented in [14], and especially, a brief review on Indic handwritten script identification in [15]. In a similar way, very recently, one can find the use of both features to separate handwritten and printed text [16]. Based on the analysis of the above techniques, we understand that, aforementioned methods have their own merits and demerits. Explicitly, local features are script dependent, sensitive to noise, skew, segmentation and are slower in computation. But, they are efficient in extracting dominant features, particularly, directional strokes of small size images [9], for instance, connected components. Global features are efficient in characterizing large size texture patterns, for example, text blocks. They are robust to noise, small skew, and are faster in computation. However, they are weak in extracting directional energies of small size images, connected components, for instance. In particular, the DCT and wavelet features employed in [1] for classifying text blocks are not potential to sustain their performance in case of word image. The global method also suffers with the issues like time complexity, segmentation, and image size. These quick observations indicate that global method is the better choice than the local [14]. Therefore, in this paper, we have presented a global technique based on directional DCT which is efficient to recover the above limitations.

The paper is organized as follows. We start by detailing the proposed approach in Section II. It mainly includes feature extraction techniques and classifier. Full Experimental results are reported in Section III, where result analysis in accordance with the scripts employed is provided. In Section IV, the paper is concluded.

\section{PROPOSED METHOD}

The primary aim is to perform word-level script identification. Words are first extracted from each document based on the morphological operators where we are primarily employed connected component analysis. The extracted words then represented with DCT features and its variant. For classification, two different well-known classifiers: LDA and $K-\mathrm{NN}$ are employed. The preliminary work is provided in [17]. 
(a)

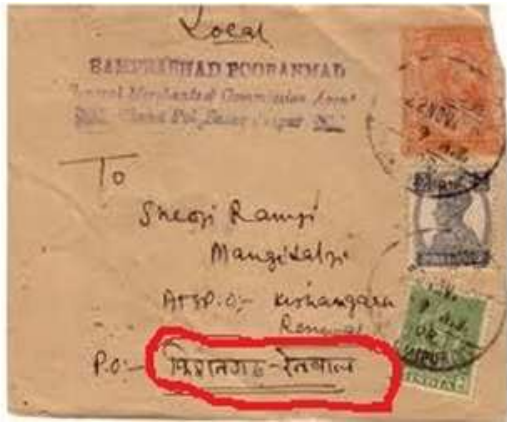

(b)

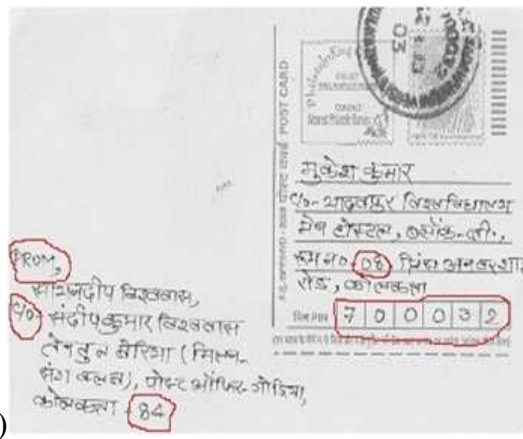

(c)

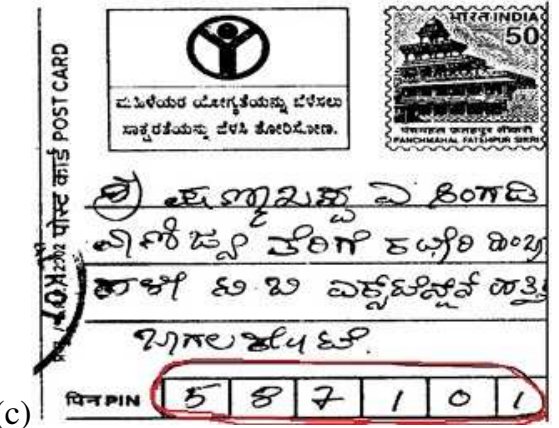

Fig. 1. A few of examples of bi-scripts' sample images: real Indian post card addresses can be written using bi-scripts, and are highlighted through red color marks.

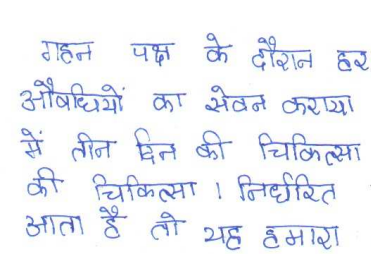

(a) Input image

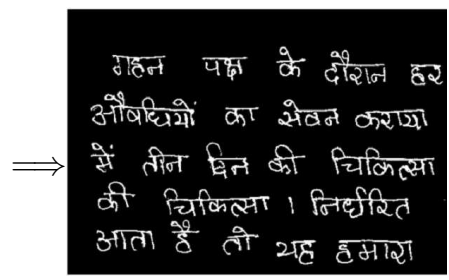

(b) Binarization

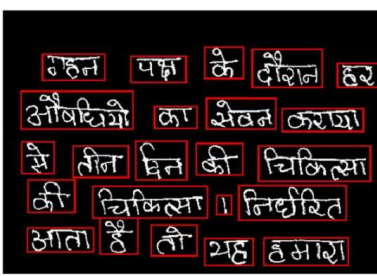

(d) Output $\Downarrow$

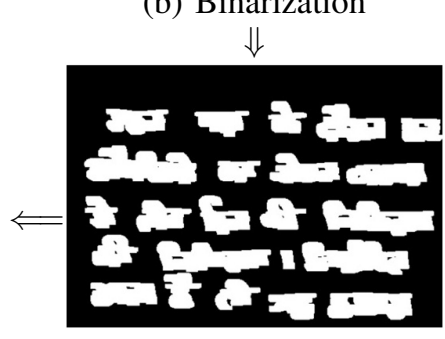

(c) Dilation
Fig. 2. An example showing word segmentation using Devanagari text block.

\section{A. Pre-processing}

The task of extraction of words from input document image is done in three steps. First, we binarized image using Otsus gray level threshold selection method [18]. Further, basic morphological operators are employed to remove the noise and special symbols from documents such as double quotations, commas etc. Then, horizontal and vertical dilation is performed using line structuring element to make each word as a single connected component.The length of the structuring element is adoptive to the script of the document. Later, bounding boxes are fixed on each component. Eventually, these connected components are extracted by applying connected component rule. The accuracy of segmentation technique is $100 \%$, except in case of touched lines/words and avoids line segmentation. The complete process of words extraction from Devanagari document can be visualized in Fig. 2.

\section{B. Features}

Discrete cosine transform (DCT) Basics. DCT basically expresses a sequence of real data points into its real spectrum. The distribution of coefficients of DCT depends on the nature of the studied image. For an image having low intensity with low spatial information, DCT provides good energy compaction in low frequency zones. In other words, conventional DCT coefficient distributions diminish as we go to higher frequencies [19]. However, the distribution of coefficients of DCT spreads over low and high frequency zones, when it is applied on images with progressive high frequency and of spatial content. There are two kinds of DCTs in use: 1) 1DDCT and 2) 2D-DCT. The 2D-DCT is a straightforward form of 1D-DCT and hereafter, we refer 2D-DCT as conventional DCT. The most common definitions of 1D-DCT and 2D-DCT are respectively as

$$
C(u)=\alpha(u) \sum_{x=0}^{N-1} f(x) \cos \left[\frac{\pi(2 x+1) u}{2 N}\right],
$$

where $u=0,1,2, \ldots, N-1$, and

$$
C(u, v)=\alpha(u) \alpha(v) \sum_{x=0}^{N-1} \sum_{y=0}^{N-1} f(x, y) \eta \rho
$$

where $\eta=\cos \left[\frac{\pi(2 x+1) u}{2 N}\right]$ and $\rho=\cos \left[\frac{\pi(2 y+1) v}{2 N}\right]$,

for $u, v=0,1,2, \ldots, N-1, \alpha(u)$ and $\alpha(v)$ are defined as

$$
\alpha(u)=\left\{\sqrt{\frac{1}{N}}, \text { for } u=0, \sqrt{\frac{2}{N}}, \text { for } u \neq 0 .\right.
$$

Directional DCT. Most of the Indian script characters are dominated by horizontal, vertical edges, holes and circular shape structures. To detain these properties, conventional DCT is not efficient. Therefore, we have motivated to formulate directional DCT to emphasize on edge information in addition to horizontal and vertical directions. To do so, we present two approaches, one by decomposing an image into left and right diagonals, and another by decomposing the DCT coefficient matrix into left and right diagonals.

Diagonally Decomposed Image (DDI). Here, each input word image matrix is converted into a square matrix by appending zeros in case of non square matrix. Then, 1D-DCTs are computed on each left and right diagonals. The complete process of feature extraction is summarized in the following paragraph.

Let $A$ be the square matrix of input image of size $N \times N$. Let the principal diagonal of $A$ be $\delta$. Let $\beta$ and $\alpha$ be N-2 upper and lower diagonals of $A$ respectively. Then the computation of six features which are denoted by $f 1, \ldots, f 6$ is discussed below. Firstly, extracted a principal diagonal $\delta$ of $A$ and computed its 
1D-DCT and then standard deviation $\sigma_{1}$ using

$$
C(u)=\alpha(u) \sum_{\mu=0}^{N-1} f(\delta) \cos \left[\frac{\pi(2 \delta+1) u}{2 N}\right],
$$

for $u=0,1,2, \ldots, N-1$ and $\mathrm{N}$ is the number of pixels in $\mu$,

$$
\sigma_{1}=\sqrt{\frac{1}{n-1}} \sum_{u=1}^{n}\left(C(u)-\overline{C(u))^{2}}\right.
$$

where $u=1,2, \ldots, n$ and $\mathrm{n}$ is the number of DCT coefficients in $\mathrm{C}(\mathrm{u})$. The standard deviation $\sigma_{1}$ is a scalar value. Secondly, $\beta$ number of upper right diagonals of $A$ are extracted and computed their 1D-DCTs as mentioned in Eq. 4. The standard deviations of $\beta$ diagonals are obtained using

$$
\sigma_{\beta}=\sqrt{\frac{1}{n-1}} \sum_{u=1}^{n}\left(C(u)_{\beta}-C(\bar{u})_{\beta}\right)^{2},
$$

where $u=1, \ldots, n$ and $\beta=1, \ldots, N-2 . \sigma_{\beta}$ is a column vector of size $N-2 \times 1$. Then, by appending the value of $\sigma_{1}$ and a zero into $\sigma_{\beta}$, we get first feature $f 1$ of dimension $N \times 1$. Similarly, $\alpha$ number of lower left diagonals of $A$ are extracted and computed their 1D-DCTs as mentioned in Eq. 1. The standard deviations of $\alpha$ diagonals are obtained using

$$
\sigma_{\alpha}=\sqrt{\frac{1}{n-1}} \sum_{u=1}^{n}\left(C(u)_{\alpha}-C(\bar{u})_{\alpha}\right)^{2},
$$

where $m=1, \ldots, N-2$ and $\sigma_{\alpha}$ is a column vector of size $N-2 \times 1$. By appending two zeros into $\sigma_{\alpha}$, we get second feature $f 2$ of dimension $N \times 1$. In this way, features $f 3$ and $f 4$ are computed by flipping the input matrix $A$. The flipped matrix is denoted by $A^{f}$ and upper, lower and principal diagonals are by $\beta^{f}, \alpha^{f}$ and $\delta^{f}$ respectively. Finally, standard deviations of 1D-DCT coefficients of horizontal and vertical frequency responses of $A$ are computed to obtain features $f 5$ and $f 6$ respectively. Thus, an integrated feature vector $\mathrm{F}=\left\{f 1_{N \times 1}, \ldots, f 6_{N \times 1}\right\}$ of size $N \times 6$ is formed. To reduce the dimension of the feature vector, again computed mean and standard deviations of $f 1_{N \times 1}, \ldots, f 6_{N \times 1}$ and reduced dimension $N \times 6$ to $12 \times 1$ (six means and six standard deviations) as the central tendency measure values of each word image and the same is used for classification.

Directional Discrete Cosine Transforms (D-DCT). In this method, first, we have computed 2D-DCT of a word image. Let the 2D-DCT coefficient matrix of a word image be $D$. Then, principal diagonal, upper $N-2$ and lower $N-2$ diagonals of $D$ is extracted before and after flipping $D$, and further computed their standard deviations respectively as discussed in the aforementioned paragraph. Meanwhile, we have also extracted features based on conventional DCT. In this case, its coefficient matrix is divided into four zones and standard deviation of each zone is computed. Thus, a feature vector of four features is formed.

\section{Classification}

Linear Discriminant Analysis is one of the most commonly used classification technique. It preserves class discriminating information to the higher extent by reducing dimensionality of feature space. It also maximizes separability between the classes by maximizing the ratio of between-class variance to the withinclass variance. In this paper, LDA is employed on a dataset $\mathrm{X}=\left[x_{1}, \ldots, x_{i}\right]$ of dimension $N \times 12(N=9000)$ and the samples $x_{i}$ are belongs to one of the class $C_{i}$, where $i=$ $1, \ldots, 6$. Further, the dimension of $x_{i}$ is $m \times p$, where $m=$ $1, \ldots, 1500$ and $p=1, \ldots, 12$. Then the classification function is defined as

$$
g(X)=W^{T} X,
$$

where $\mathrm{W}$ is the linear projection, and which maximizes between-class scatter

$$
S_{b}=S_{1}+S_{2}+\cdots+S_{6}=\sum_{i=1}^{6} \sum_{x \in c_{i}}^{6}\left(X-\mu_{i}\right)(X-\mu i)^{T},
$$

whereas it minimizes the within-class scatter

$$
S_{w}=\sum_{i}^{6} m_{i}\left(\mu_{i}-\mu\right)\left(\mu_{i}-\mu\right)^{T},
$$

where $\mu_{i}$ is the mean over class $C_{i}, \mu$ is the mean over all samples, and $m_{i}$ is the number of samples in class $C_{i}$. The classification of a new sample $\mathrm{x}$ of class label $\omega \in C_{i}$ is done based on the nearest neighbor classification rule. For this purpose, the Euclidean distance $d$ of $g(X)$ and the centers $V_{i}=W^{T} \mu_{i}$ in LDA space are compared

$$
\omega=\operatorname{argmin}_{1 \leq i \leq c} d\left(g(X), V_{i}\right)
$$

To comprehend the performance of LDA, another traditional classifier i.e., $K-\mathrm{NN}$ is used. Basically $K-\mathrm{NN}$ stores the training data $X$. Then finds the minimum $D$ distance between training sample $X$ and testing pattern $Y$ using

$$
D(X, Y)=\sqrt{\left(X-Y_{i}\right)^{T}\left(X-Y_{i}\right)} .
$$

\section{EXPERIMENTS}

\section{A. Datasets.}

There is no publicly available dataset of Indic script at present. Therefore, a dataset of 9000 handwritten text words of six scripts, namely Roman $(\mathscr{R})$, Devanagari $(\mathscr{D})$, Kannada $(\mathscr{K})$, Telugu $\left(\mathscr{T}_{\mathscr{E}}\right)$, Tamil $\left(\mathscr{T}_{\mathscr{A}}\right)$ and Malayalam $(\mathscr{M})(1500$ words of each script) is created. For generating a dataset, we have selected a paragraph of printed text from the various documents related to History, Arts, Medical, Religious and Science. This enables us to have more number of unlike words in dataset. The selected paragraphs of text are distributed among 20 writers belong to various professions and age groups. Each script is written by a different set of 20 writers. Each writer has written 75 words. The writers are asked to write the text provided for them on a A4 size paper. These papers are digitized by a scanner with a resolution of $300 \mathrm{dpi}$.

\section{B. Evaluation protocol}

To evaluate the performance of the method, $\mathbb{K}$-fold cross validation $(\mathrm{CV})$ has been implemented unlike traditional dichotomous classification. In $\mathbb{K}$-fold $\mathrm{CV}$, the original sample for every dataset is randomly partitioned into $\mathbb{K}$ sub-samples. Of the $\mathbb{K}$ sub-samples, a single sub-sample is used for validation, and the remaining $\mathbb{K}-1$ sub-samples are used for training. This process is then repeated for $\mathbb{K}$-folds, with each of the $\mathbb{K}$ sub-samples used exactly once. Eventually, a single value results from averaging all. In our tests, we use $\mathbb{K}=10$. 
TABLE I. BI-SCRIPT IDENTIFICATION RESULTS IN \% WITH LDA (LOWER TRIANGLE RESULTS ARE FROM DDI AND UPPER TRIANGLE ARE FROM D-DCT).

\begin{tabular}{|c|c|c|c|c|c|c|c|}
\hline Script & $\mathscr{R}$ & $\mathscr{D}$ & $\mathscr{K}$ & $\mathscr{T}_{\mathscr{E}}$ & $\mathscr{T}_{\mathscr{A}}$ & $\mathscr{M}$ & Average \\
\hline $\mathscr{R}$ & - & 99.00 & 96.23 & 96.60 & 98.90 & 97.86 & 97.72 \\
\hline $\mathscr{D}$ & 88.03 & - & 97.90 & 99.16 & 98.33 & 98.46 & 98.46 \\
\hline $\mathscr{K}$ & 92.80 & 94.83 & - & 85.73 & 95.03 & 95.66 & 92.14 \\
\hline $\mathscr{T}_{\mathscr{E}}$ & 95.50 & 94.93 & 87.50 & - & 96.03 & 98.66 & 97.35 \\
\hline $\mathscr{T}_{\mathscr{A}}$ & 97.53 & 96.80 & 92.63 & 93.00 & - & 99.06 & 99.10 \\
\hline $\mathscr{M}$ & 98.00 & 97.37 & 92.73 & 95.30 & 91.17 & - & $\mathbf{9 6 . 9 5}$ \\
\hline Average & 94.37 & 95.98 & 90.96 & 95.15 & 91.17 & $\mathbf{9 3 . 3 3}$ & - \\
\hline
\end{tabular}

TABLE II. BI-SCRIPT IDENTIFICATION (IN \%) WITH $K$-NN (LOWER TRIANGLE RESULTS ARE FROM DDI AND UPPER TRIANGLE ARE FROM D-DCT).

\begin{tabular}{|c|c|c|c|c|c|c|c|}
\hline Script & $\mathscr{R}$ & $\mathscr{D}$ & $\mathscr{K}$ & $\mathscr{T}_{\mathscr{E}}$ & $\mathscr{T}_{\mathscr{A}}$ & $\mathscr{M}$ & Average \\
\hline $\mathscr{R}$ & - & 95.57 & 92.23 & 92.47 & 97.03 & 97.20 & 94.90 \\
\hline $\mathscr{D}$ & 91.76 & - & 97.77 & 96.40 & 97.90 & 98.17 & 97.56 \\
\hline $\mathscr{K}$ & 94.8 & 98.20 & - & 84.70 & 93.93 & 93.67 & 90.77 \\
\hline $\mathscr{T}_{\mathscr{E}}$ & 96.6 & 95.80 & 94.13 & - & 95.73 & 95.30 & 95.52 \\
\hline $\mathscr{T}_{\mathscr{A}}$ & 97.26 & 97.67 & 94.40 & 96.17 & - & 97.67 & 97.67 \\
\hline $\mathscr{M}$ & 98.2 & 98.47 & 95.86 & 95.06 & 92.86 & - & $\mathbf{9 5 . 2 8}$ \\
\hline Average & 95.73 & 97.53 & 94.80 & 95.62 & 92.87 & $\mathbf{9 5 . 3 1}$ & - \\
\hline
\end{tabular}

TABLE III. TRI-SCRIPT IDENTIFICATION (IN \%) WITH LDA.

\begin{tabular}{|c|c|c|c|c|c|}
\hline Script & $\mathscr{R} \mathscr{D} \mathscr{K}$ & $\mathscr{R} \mathscr{D} \mathscr{T}_{\mathscr{E}}$ & $\mathscr{R} \mathscr{D} \mathscr{T}_{\mathscr{A}}$ & $\mathscr{R} \mathscr{D} \mathscr{M}$ & Average \\
\hline DDI & 85.38 & 86.37 & 89.42 & 89.44 & $\mathbf{8 7 . 6 5}$ \\
\hline D-DCT & 95.56 & 95.76 & 97.76 & 96.60 & $\mathbf{9 6 . 4 2}$ \\
\hline
\end{tabular}

TABLE IV. TRI-SCRIPT IDENTIFICATION (IN \%) WITH $K$-NN.

\begin{tabular}{|c|c|c|c|c|c|}
\hline Script & $\mathscr{R} \mathscr{D} \mathscr{K}$ & $\mathscr{R} \mathscr{D} \mathscr{T}_{\mathscr{E}}$ & $\mathscr{R} \mathscr{D} \mathscr{T}_{\mathscr{A}}$ & $\mathscr{R} \mathscr{D} \mathscr{M}$ & Average \\
\hline DDI & 90.51 & 91.38 & 92.27 & 93.11 & $\mathbf{9 1 . 8 2}$ \\
\hline D-DCT & 92.18 & 92.00 & 94.89 & 95.29 & $\mathbf{9 3 . 5 9}$ \\
\hline
\end{tabular}

\section{Results and Analysis}

The bi-script identification results are presented at lower left triangle of Table I and II, computed based on DDI method using LDA and $K$-NN classifiers respectively. The performance of $K-\mathrm{NN}$ is notable in classifying five bi-scripts with an average accuracy of $95.31 \%$, whereas LDA is $93.33 \%$. It also shows promising performance in classifying bi-script combinations such as Roman-Devanagari, Roman-Kannada, RomanTelugu, Roman-Tamil, and Roman-Malayalam with an average identification accuracy as $95.73 \%$ as compared to $94.37 \%$ of LDA (see first column of Table I and II). In case of biscript combinations, namely Devanagari-Kannada, DevanagariTelugu, Devanagari-Tamil, Devanagari-Malayalam, an average identification accuracy is $97.53 \%$ with $K-\mathrm{NN}$ and $95.98 \%$ with LDA. Tri-script and multi-script identification results of both the methods are presented in Table III, V, IV and VI. The success of $K$-NN continues with tri-script classification and showed average identification accuracy as $91.8 \%$ against $87.65 \%$ of LDA. Multi-script identification results are $86.50 \%$ and $76.00 \%$ with $K-\mathrm{NN}$ and LDA respectively. In addition, the worst performance of the LDA classifier is noticed in classifying Kannada and Telugu scripts, that is nearly $85.0 \%$ but $K$-NN showed outstanding performance of $94.13 \%$. Taken as a whole, a method of DDI with $K$-NN showed outstanding performance as compared to LDA with DDI scheme.

The results presented at upper right triangles of Table I and II are from D-DCT method, computed using LDA and $K$-NN classifiers. An average bi-script identification accuracy achieved using LDA and $K$-NN classifiers is $96.95 \%$ and 95.28\% respectively. Overall, the performance of LDA is
TABLE V. MULTI-SCRIPT IDENTIFICATION (IN \%) WITH LDA.

\begin{tabular}{|c|c|c|c|c|c|c|c|}
\hline Script & $\mathscr{R}$ & $\mathscr{D}$ & $\mathscr{K}$ & $\mathscr{T}_{\mathscr{E}}$ & $\mathscr{T}_{\mathscr{A}}$ & $\mathscr{M}$ & Average \\
\hline DDI & 80.00 & 80.33 & 66.73 & 76.33 & 73.60 & 79.00 & $\mathbf{7 6 . 0 0}$ \\
\hline D-DCT & 89.70 & 95.60 & 67.133 & 79.87 & 90.93 & 91.40 & $\mathbf{8 5 . 7 7}$ \\
\hline
\end{tabular}

TABLE VI. MULTI-SCRIPT IDENTIFICATION (IN \%) WITH $K$-NN.

\begin{tabular}{|c|c|c|c|c|c|c|c|}
\hline Script & $\mathscr{R}$ & $\mathscr{D}$ & $\mathscr{K}$ & $\mathscr{T}_{\mathscr{E}}$ & $\mathscr{T}_{\mathscr{A}}$ & $\mathscr{M}$ & Average \\
\hline DDI & 83.70 & 88.60 & 86.40 & 87.30 & 85.80 & 87.30 & $\mathbf{8 6 . 5 0}$ \\
\hline D-DCT & 83.50 & 92.40 & 77.60 & 80.30 & 91.20 & 89.10 & $\mathbf{8 5 . 7 0}$ \\
\hline
\end{tabular}

remarkable in classifying five bi-scripts and four tri-scripts combinations as compared to $K-\mathrm{NN}$. For example, tri-script average identification accuracy is $96.42 \%$ with LDA, whereas $93.58 \%$ with $K$-NN. Furthermore, the highest bi-script recognition accuracy of LDA is $99.16 \%$ for Devanagari-Telugu combination, whereas, $96.40 \%$ in case of $K-\mathrm{NN}$. In case of multi-script identification, the performance of the both the classifiers closely remains same. These observations generally show LDA is discriminant over different scripts based on DDCT features wherein $K$-NN is not. These paragraphs signify the performance of the classifiers that depends on the nature of features under study.

This paragraph emphasizes the weaknesses of the proposed algorithm. The DDI features with LDA has poor performance in discriminating between Kannada and Telugu scripts. The primary reason is shape similarity between characters between the scripts. Both the classifiers with D-DCT features do not provide efficiency in classifying (Table I and II): RomanKannada and Roman-Telugu bi-script combinations in comparison to other combinations. It is due to the writing style of the writer. The native Kannada writer has written Roman script. Therefore, writing style of Kannada is imitated while writing Roman scripts. This is an interesting observation, which is experimentally validated, by performing an experimentation with IAM dataset of Roman script (which are not written by Indian writers). As an example, we have computed horizontal features of 20 text words of IAM database and Kannada script. Meanwhile, horizontal features of 20 text words of Indian Roman ( i.e., Roman script written by Indians) and Kannada script. These are illustrated through plots (cf. Fig. 3) and showed less sparability between the features of Indian Roman and Kannada script ( $c f$. Fig. 3(a)). However, it is not in the case of IAM database Roman and Kannada script (cf. Fig. 3(b)). This analysis highlights the impact of native writer's writing style while writing Roman script.

Fig. 4(a) illustrates bi-script classification accuracies, wherein we can notice the remarkable performance of DDCT compared to conventional DCT. In addition, the time complexity difference between DDI and D-DCT in extracting features can be noticed in Fig. 4(b). Furthermore, to authenticate performance of the presented method that is D-DCT, we have extended our experiment on a dataset of 22,500 printed text words used in [5]. The classification results from multiscripts in addition to Odiya $(\mathscr{O})$ script with LDA are presented in Table VII. The average identification result is $97.06 \%$, which

TABLE VII. MULTI-SCRIPT IDENTIFICATION RESUlTS OF PRINTED TEXT WORDS IN \% WITH LDA.

\begin{tabular}{|c|c|c|c|c|c|c|}
\hline Script & $\mathscr{R}$ & $\mathscr{D}$ & $\mathscr{K}$ & $\mathscr{T}_{\mathscr{A}}$ & $\mathscr{O}$ & Average \\
\hline D-DCT & 94.13 & 97.71 & 99.82 & 95.22 & 98.44 & $\mathbf{9 7 . 0 6}$ \\
\hline
\end{tabular}




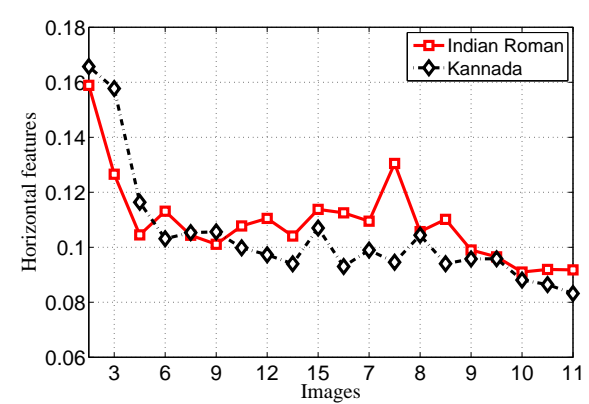

(a)

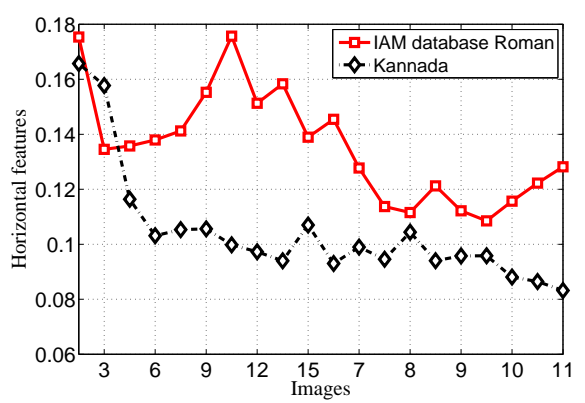

(b)

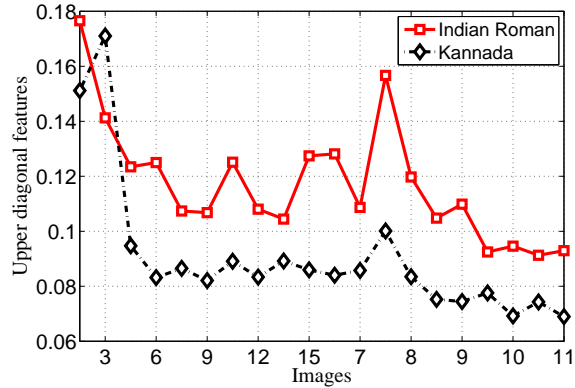

(c)

Fig. 3. An example of features spread plot (a) horizontal features of Indian Roman and Kannada script, (b) horizontal features of IAM Roman and Kannada scrip, and (c) diagonal features of Indian Roman and Kannada script.

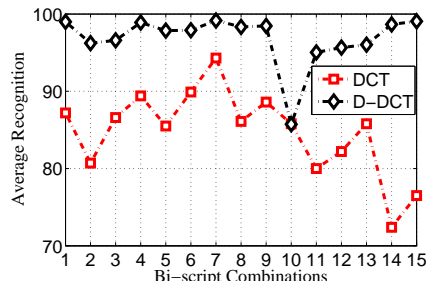

(a)

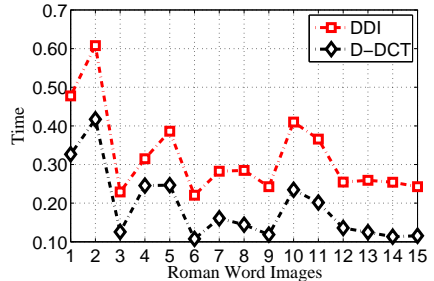

(b)
Fig. 4. It illustrates (a) script identification performance of conventional DCT and D-DCT, (b) time complexity (in sec.) of feature extraction methods (DDI and D-DCT).

is very high as compared to $93.5 \%$ and $96.0 \%$ reported with LDA and $K-\mathrm{NN}$ in [5]. The results of [5] is achieved by using 36 features; however, we achieved an accuracy of $97.06 \%$ by using only one-third features of [5] i.e., 12 features. In addition, it can be seen that diagonal features ( $c f$. Fig. 3(c)) have induced high separability between features of Indian Roman and Kannada script as compared to horizontal features (cf. Fig. 3(a)). Thus, all the above facts and figures comprehend the novelty of D-DCT plus LDA scheme.

\section{CONCLUSION AND FUtURE WORK}

In this paper, we have presented a technique of directional DCT for identifying script of handwritten text words. The novelty shown by the proposed technique in discriminating six major Indic scripts is significant. This method is based on visual perception perceived with the shapes of characters of Indic scripts, which are dominated by directional strokes. To capture these properties, we have directionally tuned the traditional DCT and achieved remarkable performance as compared to the earlier works. We have a plan to design a generalized framework to deal with all handwritten and printed Indic scripts identification task at word level. As a part of it, we are working on printed text of 11 Indic scripts used in [2] and preliminary results produced are encouraging.

\section{REFERENCES}

[1] G. Rajput and A. H.B., "Handwritten script recognition using det and wavelet features at block level," in Int. J. of Computer App., (3):158163, 2010.

[2] P. B. Pati and A. G. Ramakrishnan, "Word level multi-script identification,” Patt. Recog. Lett., 29(9):1218-1229, 2008.
[3] V. Singhal, N. Navin, and D. Ghosh, "Script-based classification of hand-written text document in a multilingual environment," in Proc. of Int. Workshop on Research Issues in Data Eng., 2003, pp. 47-54.

[4] S. B. Moussa, A. Zahour, A. Benabdelhafid, and A. Alimi, "Fractalbased system for arabic/latin, printed/handwritten script identification," in Proc. of Int. Conf. on Doc. Ana. and Recog., 2008, pp. 1-4.

[5] P. B. Pati and A. Ramakrishnan, "Hvs inspired system for script identification in indian multi-script documents," in Proc. of Int. Conf. on Doc. Ana. and Recog., 2006, pp. 380-389.

[6] K. Bhardwaj, S. Chaudhury, and S. D. Roy, "An empirical intrinsic mode based characterization of indian scripts," in Proc. of the workshop on Doc. Ana. and Recog., 2012, pp. 120-123.

[7] K. Roy, A. Banerjee, and U. Pal, "A system for word-wise handwritten script identification for indian postal automation," in Proc. of INDICON, 2004, pp. 473-480.

[8] K. Roy and U. Pal, "Word-wise hand-written script separation for indian postal automation," in Proc. of Int. Workshop on Frontiers in Handwriting Recog., 2006, pp. 521-526.

[9] M. Hangarge and B.V.Dhandra, "Offline handwritten script identification in document images," Int. J. of Computer Applications, 4(6):1-5, 2008.

[10] R. Sarkar, N. Das, S. Basu, M. Kundu, M. Nasipuri, and D. K. Basu, "Word level script identification from bangla and devanagri handwritten texts mixed with roman script," J. of Computing, 2(2):103-108, 2010.

[11] B. Alireza, K. Malike, and S. Mehdi, "A novel framework for farsi and latin script identification and farsi handwritten digit recognition," $J$. of Automatic Control, 20(1): 17-25, 2010

[12] L. Zhou, Y. Lu, and C. L. Tan, "Bangla/english script identification based on analysis of connected component profiles," in Proc. of Int. Conf. on Doc. Ana. and Recog., 2006, pp. 243-254.

[13] B. Dhandra and M. Hangarge, "Global and local features based handwritten text words and numerals script identification," in Proc. of Int. Conf. on Computational Intell. and Multimedia App., 2007, pp. 471475.

[14] D. Ghosh, T. Dube, and A. P. Shivaprasad, "Script recognition a review," IEEE Trans. on Patt. Ana. and Machine Intell., 32(12):21422161, 2010.

[15] D. Guru, M. Ravikumar, and B. H. Harish, "A review on offline handwritten script identification," in Proc. of IJCA on National Conf. on Advanced Computing and Comm., 2012, pp. 13-16.

[16] A. Belaï, K. C. Santosh, and V. P. D'Andecy, "Handwritten and printed text separation in real document," in Proc. of IAPR Conf. on Machine Vision App., 2013.

[17] M. Hangarge, K. C. Santosh, S. Doddamani, and R. Pardeshi, "Statistical texture features based handwritten and printed text classification in south indian documents," in Proc. of ICECIT, 2013, pp. 215-221.

[18] N. Otsu, "A threshold selection method from gray-level histograms," IEEE Trans. on Patt. Ana. and Machine Intell., 9(1):62-66, 1979.

[19] E. Lam and J. Goodman, "A mathematical analysis of the dct coefficient distributions for images," IEEE Trans. on Image Proces., 9(10):1661$1666,2000$. 